\title{
Sirtuin 5: a review of structure, known inhibitors and clues for developing new inhibitors
}

\author{
Lingling Yang ${ }^{1}$, Xiaobo $\mathrm{Ma}^{1}$, Yanying $\mathrm{He}^{1}$, Chen Yuan ${ }^{1}$, Quanlong Chen ${ }^{1}$, Guobo $\mathrm{Li}^{2 *}$ \& \\ Xianggui Chen ${ }^{1 * *}$ \\ ${ }^{1}$ College of Food and Bioengineering, Xihua University, Sichuan 610039, China; \\ ${ }^{2}$ Key Laboratory of Drug Targeting and Drug Delivery System of Ministry of Education, West China School of Pharmacy, Sichuan University, \\ Chengdu 610041, China
}

Received March 21,2016; accepted May 23,2016; published online November 17, 2016

\begin{abstract}
Sirtuins (SIRTs) are nicotinamide adenine dinucleotide $\left(\mathrm{NAD}^{+}\right)$-dependent protein deacetylases, which regulate important biological processes ranging from apoptosis, age-associated pathophysiologies, adipocyte and muscle differentiation, and energy expenditure to gluconeogenesis. Very recently, sirtuin 5 (SIRT5) has received considerable attention due to that it was found to have weak deacetylase activity but strong desuccinylase, demalonylase and deglutarylase activities, and it was also found to be associated with several human diseases such as cancer, Alzheimer's disease, and Parkinson's disease. In this review, we for the first time summarized the structure characteristics, known peptide and small-molecule inhibitors of SIRT5, extracted some clues from current available information and introduced some feasible, practical in silico methods, which might be useful in further efforts to develop new SIRT5 inhibitors.
\end{abstract}

Sirtuin, SIRT5 inhibitor, crystal structure, small-molecule inhibitors, computer-aided drug design

Citation: $\quad$ Yang, L., Ma, X., He, Y., Yuan, C., Chen, Q., Li, G., and Chen, X. (2017). Sirtuin 5: a review of structure, known inhibitors and clues for developing new inhibitors. Sci China Life Sci 60, 249-256. doi: 10.1007/s11427-016-0060-7

\section{INTRODUCTION}

Histone deacetylases (HDACs) are a type of enzymes that remove acetyl groups from $\varepsilon$-N-acetyl-lysine amino groups on histones and various non-histone proteins. To date, four classes of histone deacetylases have been identified in humans (Figure 1) (Hirschey, 2011; Jing and Lin, 2015). Class I, II, and IV HDACs are zinc ${ }^{2+}$-dependent deacetylases, while class III is nicotinamide adenine dinucleotide $\left(\mathrm{NAD}^{+}\right)$-dependent deacetylases. $\mathrm{NAD}^{+}$-dependent deacetylases are also termed sirtuins (SIRTs) due to their homology to the yeast silent information regulator 2 (SIR2). There

*Corresponding author (email: liguobo@scu.edu.cn)

**Corresponding author (email: chenxianggui@tom.com) are seven homologues of sirtuins in human, SIRT1 through SIRT7. SIRT1-3 have been well studied for decades (Bell and Guarente, 2011; Donmez and Outeiro, 2013; Mellini et al., 2015), whereas SIRT4-7 have received relatively little attention until recently. SIRT5 has become an active topic of research towards its biological and physiological functions (Du et al., 2011; Nishida et al., 2015; Polletta et al., 2015; Tan et al., 2014). Several studies have demonstrated that SIRT5 actually has relatively weak deacetylase activity comparing with other sirtuins. In contrast, SIRT5 has robust desuccinylation, demalonylation and deglutarylation activities in vitro and in vivo, $\sim 1,000$-fold higher catalytic efficiency than deacetylation activity (Roessler et al., 2015). Many studies also revealed that SIRT5 plays crucial roles in the regulation of ammonia detoxification (Nakagawa et al., 2009; Polletta 


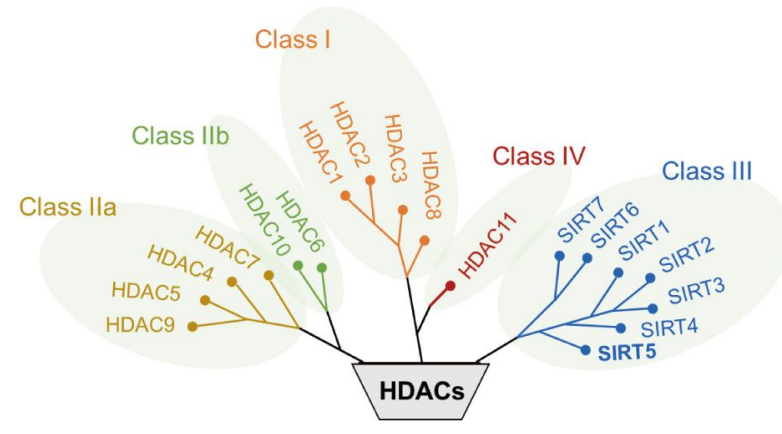

Figure 1 Phylogenetic tree of human HDAC family based on the similarity of their amino acid sequences. The HDAC family consists of four classes (Class I, II, III, and IV, shown in different colors). Class I, II, and IV HDACs are zinc $^{2+}$-dependent deacetylases, while class III HDACs are nicotinamide adenine dinucleotide $\left(\mathrm{NAD}^{+}\right)$-dependent deacetylases, called sirtuins.

et al., 2015), fatty acid oxidation (Park et al., 2013; Zhang et al., 2015), cellular respiration (Li et al., 2015a; Park et al., 2013), ketone body formation (Rardin et al., 2013), and reactive oxygen species (ROS) management (Lin et al., 2013), and disregulation or uncontrolled activation of SIRT5 can cause several human diseases, for instance cancer, Alzheimer's disease, and Parkinson's disease (Kumar and Lombard, 2015; Lai et al., 2013; Liu et al., 2015; Lu et al., 2014; Parihar et al., 2015).

Currently, the biological functions and therapeutic possibilities of SIRT5 have been well documented (Du et al., 2011; Nakagawa et al., 2009; Rardin et al., 2013; Yang et al., 2015). In this review, we present a summary of characteristics of SIRT5 structure and inhibitors, and more specifically extract some clues from current available information and suggest some useful methods for the design of new SIRT5 inhibitors, which were expected to be helpful for developing new SIRT5 inhibitors for the treatment of related diseases.

\section{STRUCTURE CHARACTERISTICS OF SIRT5}

Several high-resolution crystal structures of SIRT5 have been reported recently (Du et al., 2011; Roessler et al., 2014; Schuetz et al., 2007; Szczepankiewicz et al., 2012; Zhou et al., 2012), which provide valuable clues for the bio-functional mechanism research as well as inhibitor design. From available crystal structures, we can see that SIRT5 consists of $14 \alpha$ helices and $9 \beta$ strands, which organized two main domains: zinc ${ }^{2+}$-binding domain and Rossmann fold domain (Figure 2). The zinc ${ }^{2+}$-binding domain contains a small, three-stranded antiparallel $\beta$ sheet $(\beta 4, \beta 5$, and $\beta 6)$ and five small $\alpha$ helices $(\alpha 3, \alpha 4, \alpha 5, \alpha 8$, and $\alpha 9)$. The zinc ion, which is coordinated to four cysteine amino residues (including Cys166, Cys169, Cys207, and Cys212), is the key factor to keep the three-stranded antiparallel $\beta$ sheet stable (Figure 2). The Rossmann fold domain consists of 6 parallel $\beta$ strands $(\beta 1, \beta 2, \beta 3, \beta 7, \beta 8$ and $\beta 9)$ that form a $\beta$ sheet center, and

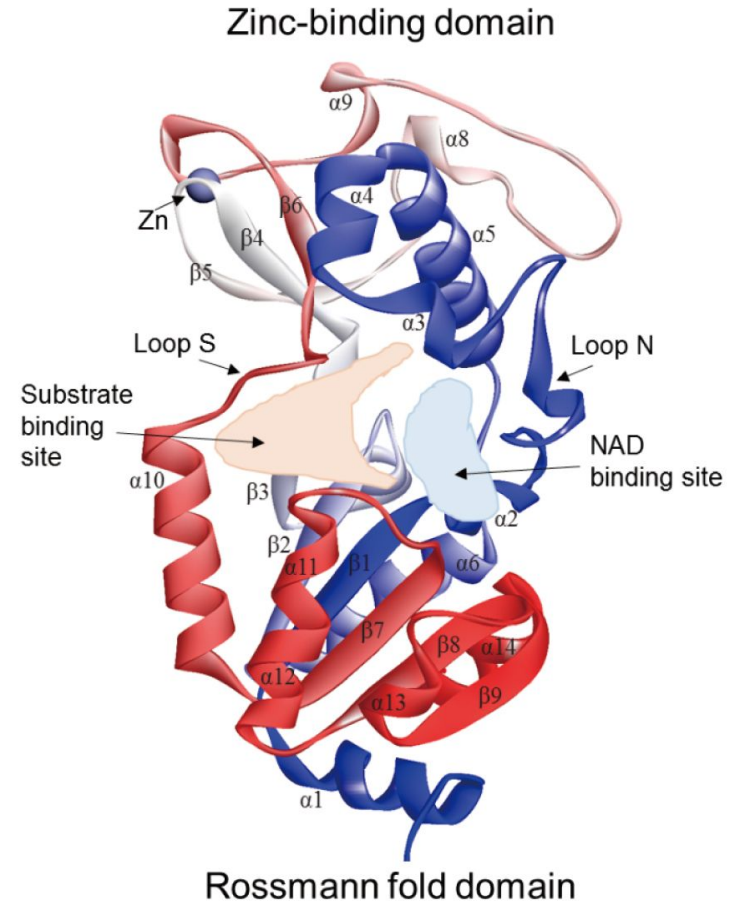

Figure 2 The overall structure of SIRT5.

9 helices $(\alpha 1, \alpha 2, \alpha 6, \alpha 7, \alpha 10, \alpha 11, \alpha 12, \alpha 13$ and $\alpha 14)$ that surround the $\beta$ sheet center. The cleft between these two domains is connected by several loops, which actually forms the catalytic site, including substrate binding site and $\mathrm{NAD}^{+}$binding site (Figure 2). The loop S, which connects the strand $\beta 6$ of the zinc binding domain and the helix $\alpha 10$ of Rossmann fold domain, is very important for substrate binding. The loop N, which connects the helix $\alpha 3$ of the zinc ${ }^{2+}$ binding domain and the $\alpha 2$ helix of the Rossmann fold domain, is important for $\mathrm{NAD}^{+}$binding (Figure 2).

By comparing crystal structures of SIRT5 with other sirtuins including SIRT1 (Davenport et al., 2014), SIRT2 (Moniot et al., 2013), SIRT3 (Jin et al., 2009), and SIRT6 (Pan et al., 2011), SIRT5 was observed to have a similar overall domain organization and fold to the structures of SIRT1, SIRT2, and SIRT3 (Figure 3A), but a different domain fold with SIRT6 especially in zinc ${ }^{2+}$ binding domain and catalytic site (Figure 3B). As shown in Figure 3A, the catalytic sites of SIRT5, SIRT1, SIRT2, and SIRT3 superimpose very closely, and the residues around the catalytic site are relatively conserved. For example, the residues Phe223, Leu227, and Val254 in SIRT5 can be found at the corresponding position in SIRT1, SIRT2, and SIRT3 (Figure 3A). These three hydrophobic residues form a small triangle and define the entrance for the acyl-lysine group of the substrate. Especially, Phe223 seems to be a gate keeper, which can block big motifs enter the substrate binding site and make way for kind of linear residues such as lysine, hence dramatically maintaining the substrate selec- 
A

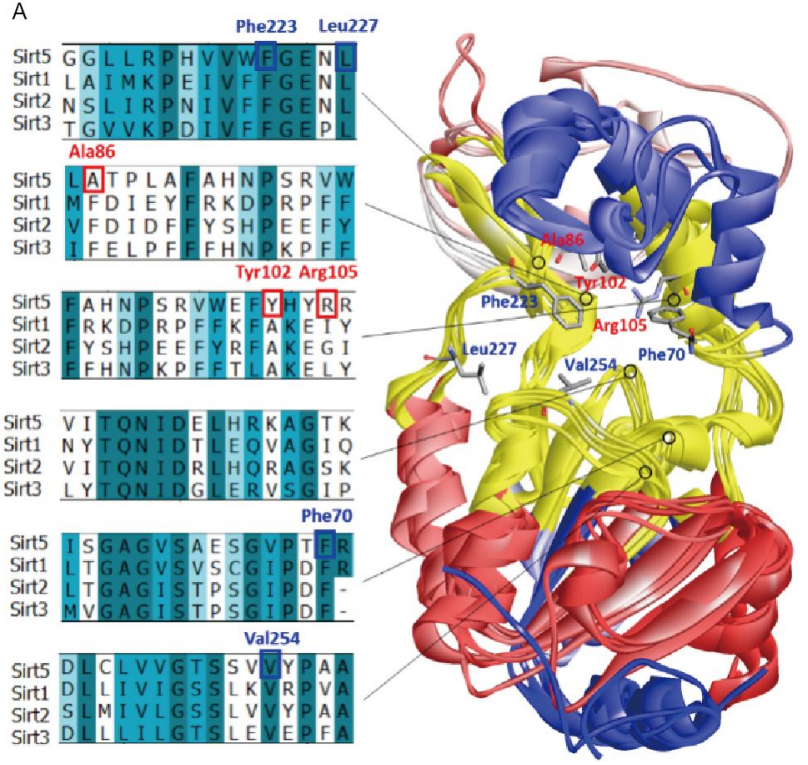

B

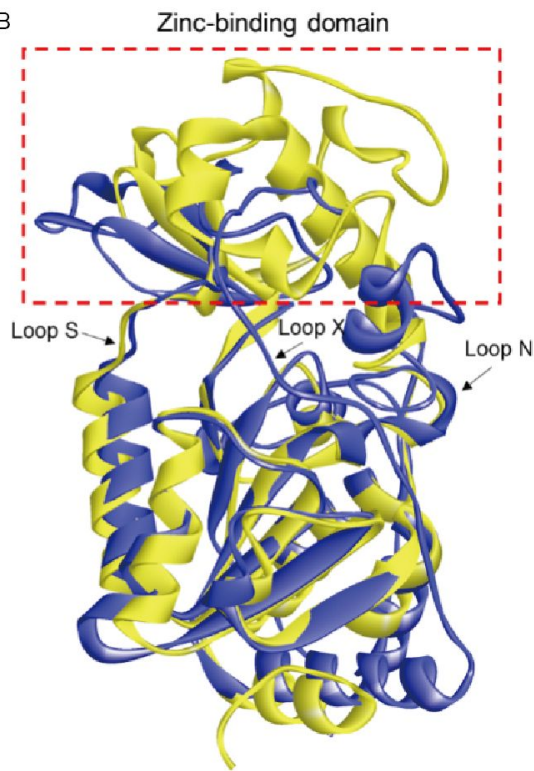

Figure 3 Bioinformatics analyses revealed that SIRT5 has a similar overall domain organization and fold to SIRT1, SIRT2, and SIRT3, but different to SIRT6 especially in zinc-binding domain and catalytic site. A, Comparative analysis of SIRT5 with SIRT1, SIRT2, and SIRT3. The catalytic sites of these sirtuins are highlighted in yellow. B, Comparing SIRT5 with SIRT6. SIRT5 is shown in yellow, and SIRT6 is highlighted in blue.

tivity. Another important residue Phe70 is also highly conserved within sirtuin family (Figure 3A). Phe70 was found to have two different orientations, like a switch residue. When the benzene ring of Phe 70 interacts with the N-ribose ring via pi-hydrophobic interactions, Phe 70 can stabilize the $\mathrm{NAD}^{+}$or intermediate, but whilst Phe70 is perpendicular to the ribosyl ring of $\mathrm{NAD}^{+}$, it seems to favor nicotinamide escape. There also exist many other conserved residues in sirtuin members (Figure 3A), which make them possess similar biological functions. In contrast, an obvious difference between the domain folds of SIRT5 and SIRT6 was observed (Figure 3B); SIRT6 does not have small $\alpha$ helices in the zinc ${ }^{2+}$-binding domain, which is the conserved structure characteristic for SIRT1, SIRT2, SIRT3 and SIRT5 (Figure 3A). Besides, SIRT6 possesses a specific loop X located outside of the binding site leading to a different active site compared with SIRT5, which is an important factor contributing to its specific substrate profile (Figure 3B).

Structural differences between sirtuins can be observed in the variable amino- and carboxy-terminal extensions (Figure $3 \mathrm{~A}$ ), where they may regulate their subcellular localizations and catalytic activities, and keep their own specific biological and physiological functions. Even though in core catalytic domain, SIRT5 possesses its own specific residues. For example, the two non-hydrophobic residues Tyr102 and $\operatorname{Arg} 105$, located in the deep end of substrate binding pocket, are exclusive to SIRT5 (Figure 3A), which are likely to specifically recognize negatively charged acyl-lysine groups (i.e. succinyl, demalonyl, and glutaryl). SIRT5 has a small residue Ala86 (Phenylalanine residue is in the corresponding position to SIRT1, SIRT2, and SIRT3, see Figure 3A), which make SIRT5 harbor a larger acyl-lysine binding pocket than other sirtuins. Due to these specific structural characteristics, SIRT5 is the only sirtuin member to date demonstrated to have efficient demalonylase and desuccinylase activity (Du et al., 2011).

To further understand how substrate and $\mathrm{NAD}^{+}$bind and the specificity of SIRT5, one crystal structure reported recently by Du et al. (PDB code: 3RIY) was taken for example (Du et al., 2011). This is a ternary crystal structure, in which co-factor $\mathrm{NAD}^{+}$and succinyl-H3K9 substrate peptide bind. As shown in Figure 4, substrate peptide and $\mathrm{NAD}^{+}$both locate in the interspace between zinc ${ }^{2+}$ binding domain and Rossmann fold domain. Those residues including Phe70, Arg71, Gln140, Asn141, and Asp143, are very important for $\mathrm{NAD}^{+}$ binding (Figure 4). The Gln140 and Asn141 hold the binding of $\mathrm{NAD}^{+}$ribose part, and Asp143 directly interacts with nicotinamide part. More interestingly, the flexible residue Phe70 plays a crucial role in keep $\mathrm{NAD}^{+}$binding/unbinding, like a switch residue (Figure 4). For substrate peptide binding, the important residues include His158, Phe223, Try 102 and Arg105 (Figure 4). As described above, Tyr102 and Arg 105 are the unique residues for SIRT5, which form hydrogen-bonding and ionic-bonding interactions with the carboxyl of succinyl-lysine substrate. Besides, we observed that the succinyl moiety of substrate peptide is positioned to make direct interactions with ribose motif of $\mathrm{NAD}^{+}$.

\section{SIRT5 INHIBITORS}

SIRT5 is known to be involved in cellular metabolism and metabolic energy homeostasis by its various protein substr- 


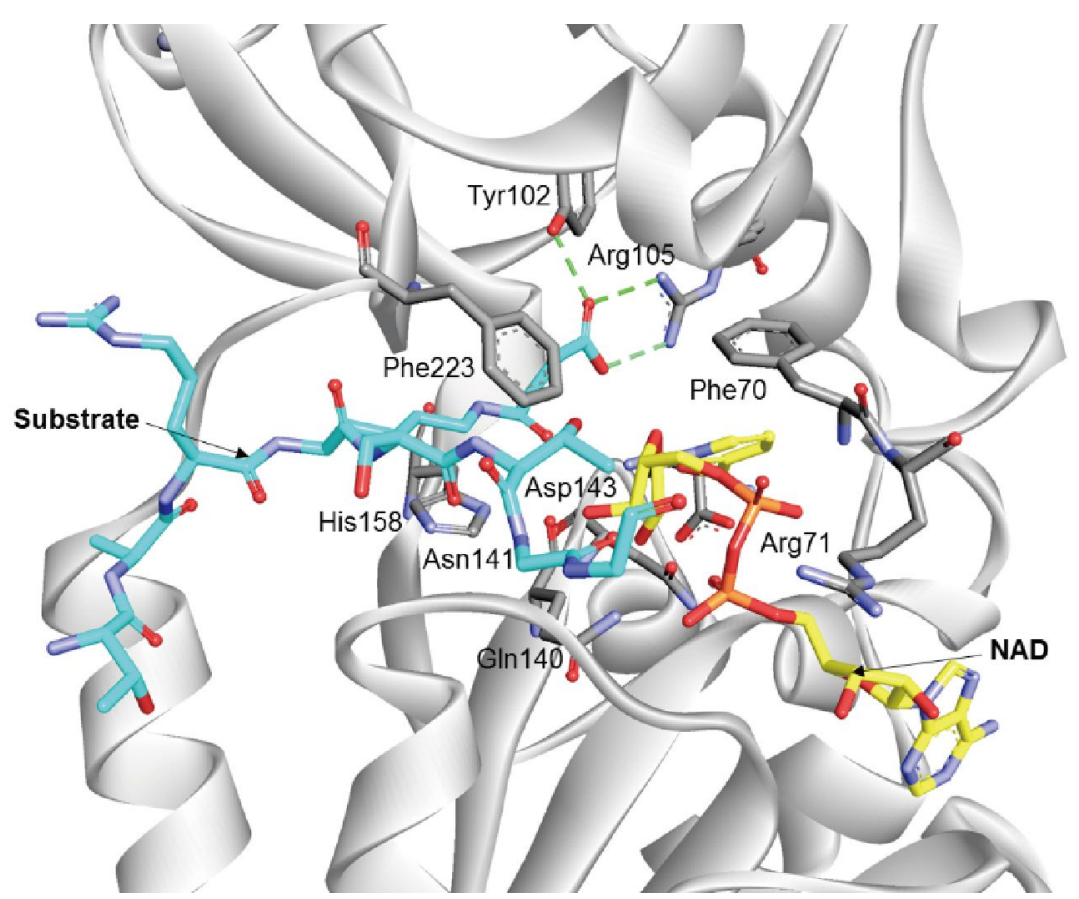

Figure 4 An example of the succinyl-K9 peptide-NAD ${ }^{+}$ternary crystal structure (PDB code: $\left.3 R I Y\right)$.

strates. The possibilities of targeting SIRT5 are beginning to be more extensively investigated with aim of providing new strategies for the treatment of related diseases, since SIRT5 was demonstrated to be activation/over-expression in several human diseases (i.e. cancer). For example, an integrated genomic analyses of ovarian carcinoma revealed that region encompassing the SIRT5 locus was amplified 30\% in ovarian carcinoma (Network, 2011). Very recently, Lu et al. found that SIRT5 is over-expressed in advanced non-small cell lung cancer (NSCLC), and knock-down of SIRT5 could repress the growth rate of NSCLC cell lines (Lu et al., 2014). Lutz and his colleagues revealed that the expression of SIRT5 was increased during the progression of Alzheimer's disease in contrast to SIRT1 and SIRT (Lutz et al., 2014). Glorioso et al. found that SIRT5 might be an important risk factor contributing to mitochondrial dysfunction-related diseases including Parkinson's disease through accelerating molecular aging of disease-related genes (Glorioso et al., 2011). Discovery of SIRT5 inhibitors is likely to be an attractive area for treatment of associated diseases. The reported peptide and small-molecule inhibitors of SIRT5 are hence summarized in following section.

\section{Peptide inhibitors}

Peptide inhibitors usually can be used as not only chemical probes for molecular mechanism research but also good starting points for small-molecule inhibitor design. Inspired by the fact that thioacetyl peptides can inhibit the deacetylase activities of sirtuins via forming a stable covalent intermediate, He and co-workers (He et al., 2012) synthesized the first SIRT5-specific inhibitor, namely histone H3K9 thiosuc- cinyl peptide (H3K9TSu, Figure 5), which is a competitive

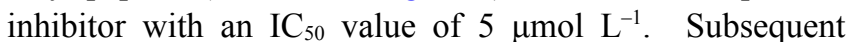
structure-activity relationship (SAR) study showed that the peptide inhibitors with a thiosuccinyl-lysine residue at the $\mathrm{C}$-terminus or $\mathrm{N}$-terminus were less potent than that in the middle, and the longer thiosuccinyl peptide seems to have better inhibitory activity against SIRT5. Another study by Roessler et al. investigated SAR of lysine side chain of SIRT5 substrate carbamoyl phosphate synthetase (CPS1), and eventually identified several potent peptide inhibitors 1-4 (Figure 5) (Roessler et al., 2014), of $\mathrm{K}_{\mathrm{i}}$ values are $17.2 \pm 1.31,38.1 \pm 0.63,4.3 \pm 0.32$, and $10.6 \pm 0.66 \mu \mathrm{mol}$ $\mathrm{L}^{-1}$, respectively. Very recently, Zang et al. identified a selective, potent SIRT5 inhibitory warhead, namely $\mathrm{N}^{\varepsilon}$-carboxyethyl-thiourea-lysine, and compound 5 harboring this warhead has an $\mathrm{IC}_{50}$ value of $5.0 \mu \mathrm{mol} \mathrm{L} \mathrm{L}^{-1}$ against SIRT5 (Figure 5) (Zang et al., 2015). More interestingly, peptide inhibitors containing this warhead seem to circumvent the cytotoxicity problem associated with the inhibitors bearing the $\mathrm{N}^{\varepsilon}$-thioacyl-lysines.

\section{Small molecule inhibitors}

Given the fact that peptide inhibitors generally suffered from limited pharmacological relevance due to their size, limited biostability and poor membrane permeability, it is desirable to develop small-molecule SIRT5 inhibitors for characterizing the physiological function and therapeutic potential. Suramin is one of the very few known pharmacological SIRT5 smallmolecule inhibitors, which inhibits SIRT5 non-specifically at submicromolar level by blocking substrate and $\mathrm{NAD}^{+}$binding (Figure 6) (Gertz and Steegborn, 2010; Schuetz et al., 2007). 


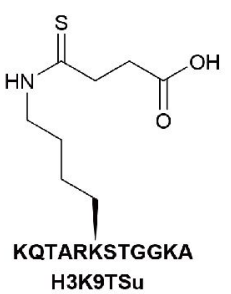
H3K9TSu

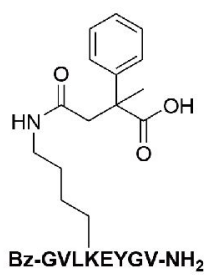

3

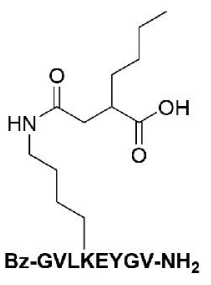

1
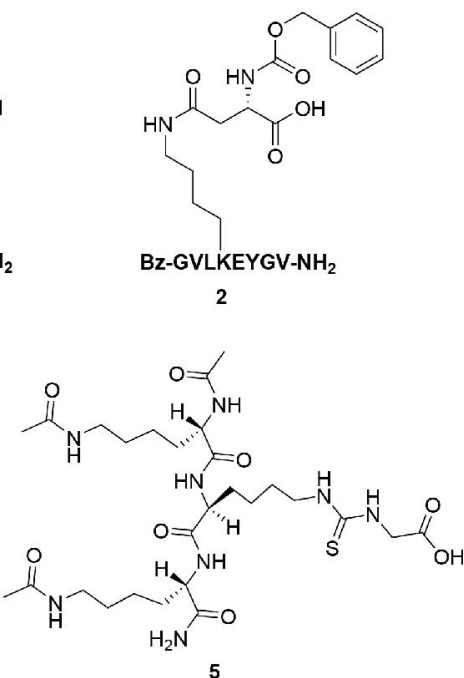

Figure 5 The known peptide inhibitors of SIRT5.<smiles></smiles><smiles>NC(=O)c1cccnc1</smiles>

Nicotinamide<smiles>O=C1Nc2ccc(I)cc2/C1=C\c1cc(Br)c(O)c(Br)c1</smiles>

GW5074
Suramin<smiles>CC(NC(=O)c1ccccc1/N=C/c1c(O)ccc2ccccc12)c1ccccc1</smiles>

Sirtinol<smiles>O=c1[nH]c(-c2ccccc2)c(Cc2c(O)ccc3ccccc23)c(=O)[nH]1</smiles>

Cambinol<smiles>O=C1NC(=S)NC(=O)C1=Cc1cn(Cc2ccccc2)c2ccccc12</smiles>

9<smiles>O=C1NC(=S)NC(=O)C1=Cc1ccc(OCc2ccccc2)cc1</smiles>

10

Figure 6 The known small-molecule inhibitors of SIRT5.

Maurer et al. using their own established method tested several known SIRT1-3 small-molecule inhibitors and co-products of the reaction of deacetylation and deacylation, nicotinamide against SIRT5, and found that sirtinol, cambinol and nicotinamide (Figure 6) can inhibit SIRT5 at submicromolar level (Maurer et al., 2012). Similarly, Suenkel's group has found that GW5074 (Figure 6) has considerable inhibitory activity to SIRT5's desuccinylation activity ( $85 \%$ inhibitory activity at $\left.100 \mu \mathrm{mol} \mathrm{L}{ }^{-1}\right)$, but it has weaker effects to SIRT5 deacetylation (Suenkel et al., 2013). Different from above studies, Maurer et al. identified thiobarbiturate-containing compound 9 and compound 10 (Figure 6) as new inhibitors by direct screening, which have low $\mu \mathrm{mol} \mathrm{L}^{-1}$ potency against
SIRT5 (Maurer et al., 2012). These inhibitors should be good starting points to develop new SIRT5 inhibitors.

\section{CLUES AND METHODS FOR THE DESIGN OF NEW SIRT5 INHIBITORS}

As mentioned above, SIRT5 is a potential drug target, but few SIRT5 inhibitors have been reported so far. It is presently desirable to discover new SIRT5 inhibitors for molecular mechanism research and drug discovery. Some clues extracted from available crystal structures and inhibitors of SIRT5 as well as those of other sirtuins, and practical computer-aided drug design (CADD) methods given in following 
section should be useful in further efforts to develop new SIRT5 inhibitors.

The $\mathrm{NAD}^{+}$binding site and substrate binding site are currently two main binding pockets for SIRT5 inhibitors. For $\mathrm{NAD}^{+}$binding site, those attempts including identifying $\mathrm{NAD}^{+}$analogues and competitive inhibitors could be an effective strategy to inhibit SIRT5. However, due to that a large number of human enzymes use $\mathrm{NAD}^{+}$as a co-substrate, the inhibitors against $\mathrm{NAD}^{+}$binding site may result in concerns of their target selectivity. In contrast, to discover selective SIRT5 inhibitors via targeting substrate binding site is likely to be a recommended strategy since some specific residues (i.e. Tyr102, Arg105, and Ala86) form a relatively large, specific substrate binding pocket (for details please see structure characteristics of SIRT5).

According to available substrate-SIRT5 complex structures, three important features for substrate binding concluded here could be useful for designing inhibitors targeting substrate binding pocket. First, two specific non-hydrophobic residues, Arg 105 and Tyr102, in the deep end of substrate binding pocket interact with the succinyl-lysine group of substrate via hydrogen-bonding and electrostatic interactions (Figure 4), indicating that negatively charged groups (i.e. carboxyl and tetrazole) or polar chemical groups (i.e. sulfonamide and isoxazol-3-ol) could be preferred chemical moieties to occupy this kind of pocket. Second, Phe223 in loop $\mathrm{S}$ is likely to be a gate keeper to restrict large fragments to go into the deep end of substrate binding pocket, suggesting that it is desirable to design some rigid, linear chemical moieties or small aromatic rings as linkage scaffolds with key pharmacophore features. Third, three hydrophobic residues including Leu227, Val254, Phe223 form a triangle-shaped entrance for substrate binding, which suggests that small-molecule compounds with similar triangle shapes might perfectly fit with the substrate binding site. Besides, some clues also could be obtained from SIRT5's neighbor family members, even though they have structural differences. For example, Rumpf et al. recently found a new binding pocket for SIRT2 induced by a selective inhibitor, termed SirReal2 (Rumpf et al., 2015). This study might inspire people to search novel SIRT5 inhibitors targeting other possible unexploited binding pockets.

Currently, a number of well-established CADD methods (Ekins et al., 2007; Li et al., 2015c) could be very useful in designing new SIRT5-specific inhibitors. For example, according to available substrate-SIRT5 crystal structures, three dimensional (3D) shape-based methods such as ROCS, ShaEP and Phase Shape would be effective tools to identify small-molecule compounds having similar shape with SIRT5 substrate. Pharmacophore-based methods (Yang et al., 2012; Yang, 2010) can be used to generate a reasonable pharmacophore model from substrate-SIRT5 structures to search for potential inhibitors. Molecular docking and molecular dynamics methods also are very useful in lead discovery and lead optimization. Recently, some combined hierarchical in silico methods were established, which could avoid inherent shortcomings of each individual method and capitalize on their mutual strengths (Li et al., 2011; Ren et al., 2011). A hierarchical virtual screening method described in following might be a useful method to identify new SIRT5 inhibitors. The first step is to establish a carboxyl/carboxyl isostere focused chemical library for SIRT5 since carboxyl/carboxyl isostere groups may form interactions with the specific residues Arg105 and Tyr102. The second step is to use molecular docking to search possible binding poses for the established focused chemical library. The third step is to use interaction fingerprints-based methods or pharmacophore-based to filter the docking poses (de Graaf et al., 2011; Muthas et al., 2008). The final step is to use rescoring methods for example molecular mechanics Poisson-Boltzmann surface area (MM-PBSA) (Suri and Naik, 2015) and ID-Score (Li et al., 2013) to predict the binding affinity for providing information to help select potential hit compounds. The use of these multiple, complementary methods could increase the success rate of identifying new SIRT5 inhibitors. In addition to above described methods, structure-based or pharmacophore-based de novo design methods (Schneider and Fechner, 2005; Talamas et al., 2013) as well as scaffold hopping or substitute modification methods (Li et al., 2015b; Mauser and Guba, 2008) are worth to use because these methods might be able to discover novel SIRT5 inhibitors with novel chemical scaffolds.

\section{SUMMARY}

SIRT5 is an attractive enzyme for mechanism research since it not only has deacetylase activity but also has strong demalonylase, desuccinylase and deglutarylase activities. SIRT5 is also considered as a potential molecular target for treatment of several human diseases. In this review, we summarized the structural characteristics of SIRT5 and its structural differences to other sirtuins, and highlighted the detailed, specific structural binding of SIRT5 substrates. In addition, we gave a summary of currently known peptide and small-molecule SIRT5 inhibitors. Moreover, we extracted some clues from current available information and gave some feasible, practical in silico methods for SIRT5 inhibitor design. We hope this review can help people understand SIRT5 structure characteristics and provide useful information for further efforts to develop new SIRT5 inhibitors for related molecular mechanism research as well as drug discovery \& development.

Compliance and ethics The author(s) declare that they have no conflict of interest. 
Acknowledgements This work was supported by the Chun hui of Ministry of Education Project (Z2015120), the National Natural Science Foundation of China (81502989), and the China Postdoctoral Science Foundation Funded Project (2015M570789).

Bell, E.L., and Guarente, L. (2011). The SirT3 divining rod points to oxidative stress. Mol Cell 42, 561-568.

Davenport, A.M., Huber, F.M., and Hoelz, A. (2014). Structural and functional analysis of human SIRT1. J Mol Biol 426, 526-541.

de Graaf, C., Kooistra, A.J., Vischer, H.F., Katritch, V., Kuijer, M., Shiroishi, M., Iwata, S., Shimamura, T., Stevens, R.C., de Esch, I.J.P., and Leurs, R. (2011). Crystal structure-based virtual screening for fragment-like ligands of the human histamine $\mathrm{H}_{1}$ receptor. J Med Chem 54, 8195-8206.

Donmez, G., and Outeiro, T.F. (2013). SIRT1 and SIRT2: emerging targets in neurodegeneration. EMBO Mol Med 5, 344-352.

Du, J., Zhou, Y., Su, X., Yu, J.J., Khan, S., Jiang, H., Kim, J., Woo, J., Kim, J.H., Choi, B.H., He, B., Chen, W., Zhang, S., Cerione, R.A., Auwerx, J., Hao, Q., and Lin, H. (2011). Sirt5 is a NAD-dependent protein lysine demalonylase and desuccinylase. Science 334, 806-809.

Ekins, S., Mestres, J., and Testa, B. (2007). In silico pharmacology for drug discovery: methods for virtual ligand screening and profiling. Br J Pharmacol 152, 9-20.

Gertz, M., and Steegborn, C. (2010). Function and regulation of the mitochondrial sirtuin isoform Sirt5 in Mammalia. Biochim Biophys Acta 1804, 1658-1665.

Glorioso, C., Oh, S., Douillard, G.G., and Sibille, E. (2011). Brain molecular aging, promotion of neurological disease and modulation by sirtuin 5 longevity gene polymorphism. Neurobiol Dis 41, 279-290.

He, B., Du, J., and Lin, H. (2012). Thiosuccinyl peptides as Sirt5-specific inhibitors. J Am Chem Soc 134, 1922-1925.

Hirschey, M.D. (2011). Old enzymes, new tricks: sirtuins are $\mathrm{NAD}^{+}$-dependent de-acylases. Cell Metab 14, 718-719.

Jin, L., Wei, W., Jiang, Y., Peng, H., Cai, J., Mao, C., Dai, H., Choy, W., Bemis, J.E., Jirousek, M.R., Milne, J.C., Westphal, C.H., and Perni, R.B. (2009). Crystal structures of human SIRT3 displaying substrate-induced conformational changes. J Biol Chem 284, 24394-24405.

Jing, H., and Lin, H. (2015). Sirtuins in epigenetic regulation. Chem Rev $115,2350-2375$.

Kumar, S., and Lombard, D.B. (2015). Mitochondrial sirtuins and their relationships with metabolic disease and cancer. Antioxid Redox Signal 22, 1060-1077.

Lai, C.C., Lin, P.M., Lin, S.F., Hsu, C.H., Lin, H.C., Hu, M.L., Hsu, C.M., and Yang, M.Y. (2013). Altered expression of SIRT gene family in head and neck squamous cell carcinoma. Tumor Biol 34, 1847-1854.

Li, F., He, X., Ye, D., Lin, Y., Yu, H., Yao, C., Huang, L., Zhang, J., Wang, F., Xu, S., Wu, X., Liu, L., Yang, C., Shi, J., He, X., Liu, J., Qu, Y., Guo, F., Zhao, J., Xu, W., and Zhao, S. (2015a). NADP ${ }^{+}$-IDH mutations promote hypersuccinylation that impairs mitochondria respiration and induces apoptosis resistance. Mol Cell 60, 661-675.

Li, G.B., Ji, S., Yang, L.L., Zhang, R.J., Chen, K., Zhong, L., Ma, S., and Yang, S.Y. (2015b). LEADOPT: an automatic tool for structurebased lead optimization, and its application in structural optimizations of VEGFR2 and SYK inhibitors. Eur J Med Chem 93, 523-538.

Li, G.B., Yang, L.L., Feng, S., Zhou, J.P., Huang, Q., Xie, H.Z., Li, L.L., and Yang, S.Y. (2011). Discovery of novel mGluR1 antagonists: a multistep virtual screening approach based on an SVM model and a pharmacophore hypothesis significantly increases the hit rate and enrichment factor. Bioorg Med Chem Lett 21, 1736-1740.

Li, G.B., Yang, L.L., Wang, W.J., Li, L.L., and Yang, S.Y. (2013). ID-score: a new empirical scoring function based on a comprehensive set of descriptors related to protein-ligand interactions. J Chem Inf Model 53, 592-600.

Li, G.B., Yang, L.L., Yuan, Y., Zou, J., Cao, Y., Yang, S.Y., Xiang, R., and Xiang, M. (2015c). Virtual screening in small molecule discovery for epigenetic targets. Methods 71, 158-166.
Lin, Z.F., Xu, H.B., Wang, J.Y., Lin, Q., Ruan, Z., Liu, F.B., Jin, W., Huang, H.H., and Chen, X. (2013). SIRT5 desuccinylates and activates SOD1 to eliminate ROS. Biochem Biophys Res Commun 441, 191-195.

Liu, L., Peritore, C., Ginsberg, J., Shih, J., Arun, S., and Donmez, G. (2015). Protective role of SIRT5 against motor deficit and dopaminergic degeneration in MPTP-induced mice model of Parkinson's disease. Behav Brain Res 281, 215-221.

Lu, W., Zuo, Y., Feng, Y., and Zhang, M. (2014). SIRT5 facilitates cancer cell growth and drug resistance in non-small cell lung cancer. Tumor Biol 35, 10699-10705.

Lutz, M.I., Milenkovic, I., Regelsberger, G., and Kovacs, G.G. (2014). Distinct patterns of sirtuin expression during progression of Alzheimer's disease. Neuromol Med 16, 405-414.

Maurer, B., Rumpf, T., Scharfe, M., Stolfa, D.A., Schmitt, M.L., He, W., Verdin, E., Sippl, W., and Jung, M. (2012). Inhibitors of the NAD ${ }^{+}$-dependent protein desuccinylase and demalonylase Sirt5. ACS Med Chem Lett 3, 1050-1053.

Mauser, H., and Guba, W. (2008). Recent developments in de novo design and scaffold hopping. Curr Opin Drug Discov Devel 11, 365-374..

Mellini, P., Valente, S., and Mai, A. (2015). Sirtuin modulators: an updated patent review (2012-2014). Expert Opin Ther Patents 25, 5-15.

Moniot, S., Schutkowski, M., and Steegborn, C. (2013). Crystal structure analysis of human Sirt2 and its ADP-ribose complex. J Struct Biol 182, 136-143.

Muthas, D., Sabnis, Y.A., Lundborg, M., and Karlén, A. (2008). Is it possible to increase hit rates in structure-based virtual screening by pharmacophore filtering? An investigation of the advantages and pitfalls of post-filtering. J Mol Graph Model 26, 1237-1251.

Nakagawa, T., Lomb, D.J., Haigis, M.C., and Guarente, L. (2009). SIRT5 deacetylates carbamoyl phosphate synthetase 1 and regulates the urea cycle. Cell 137, 560-570.

Network, A.R. (2011). Integrated genomic analyses of ovarian carcinoma. Nature 474, 609-615..

Nishida, Y., Rardin, M.J., Carrico, C., He, W., Sahu, A.K., Gut, P., Najjar, R., Fitch, M., Hellerstein, M., Gibson, B.W., and Verdin, E. (2015). SIRT5 regulates both cytosolic and mitochondrial protein malonylation with glycolysis as a major target. Mol Cell 59, 321-332.

Pan, P.W., Feldman, J.L., Devries, M.K., Dong, A., Edwards, A.M., and Denu, J.M. (2011). Structure and biochemical functions of SIRT6. J Biol Chem 286, 14575-14587.

Parihar, P., Solanki, I., Mansuri, M.L., and Parihar, M.S. (2015). Mitochondrial sirtuins: emerging roles in metabolic regulations, energy homeostasis and diseases. Exp Gerontol 61, 130-141.

Park, J., Chen, Y., Tishkoff, D.X., Peng, C., Tan, M., Dai, L., Xie, Z., Zhang, Y., Zwaans, B.M.M., Skinner, M.E., Lombard, D.B., and Zhao, Y. (2013). SIRT5-mediated lysine desuccinylation impacts diverse metabolic pathways. Mol Cell 50, 919-930.

Polletta, L., Vernucci, E., Carnevale, I., Arcangeli, T., Rotili, D., Palmerio, S., Steegborn, C., Nowak, T., Schutkowski, M., Pellegrini, L., Sansone, L., Villanova, L., Runci, A., Pucci, B., Morgante, E., Fini, M., Mai, A., Russo, M.A., and Tafani, M. (2015). SIRT5 regulation of ammonia-induced autophagy and mitophagy. Autophagy 11, 253-270.

Rardin, M.J., He, W., Nishida, Y., Newman, J.C., Carrico, C., Danielson, S.R., Guo, A., Gut, P., Sahu, A.K., Li, B., Uppala, R., Fitch, M., Riiff, T., Zhu, L., Zhou, J., Mulhern, D., Stevens, R.D., Ilkayeva, O.R., Newgard, C.B., Jacobson, M.P., Hellerstein, M., Goetzman, E.S., Gibson, B.W., and Verdin, E. (2013). SIRT5 regulates the mitochondrial lysine succinylome and metabolic networks. Cell Metab 18, 920-933.

Ren, J.X., Li, L.L., Zheng, R.L., Xie, H.Z., Cao, Z.X., Feng, S., Pan, Y.L., Chen, X., Wei, Y.Q., and Yang, S.Y. (2011). Discovery of novel Pim-1 kinase inhibitors by a hierarchical multistage virtual screening approach based on SVM model, pharmacophore, and molecular docking. J Chem Inf Model 51, 1364-1375.

Roessler, C., Nowak, T., Pannek, M., Gertz, M., Nguyen, G.T.T., Scharfe, M., Born, I., Sippl, W., Steegborn, C., and Schutkowski, M. (2014). Chemical probing of the human sirtuin 5 active site reveals its substrate 
acyl specificity and peptide-based inhibitors. Angew Chem Int Engl 53, 10728-10732.

Roessler, C., Tüting, C., Meleshin, M., Steegborn, C., and Schutkowski, M. (2015). A novel continuous assay for the deacylase sirtuin 5 and other deacetylases. J Med Chem 58, 7217-7223.

Rumpf, T., Schiedel, M., Karaman, B., Roessler, C., North, B.J., Lehotzky, A., Oláh, J., Ladwein, K.I., Schmidtkunz, K., Gajer, M., Pannek, M., Steegborn, C., Sinclair, D.A., Gerhardt, S., Ovádi, J., Schutkowski, M., Sippl, W., Einsle, O., and Jung, M. (2015). Selective Sirt2 inhibition by ligand-induced rearrangement of the active site. Nat Commun 6, 6263.

Schneider, G., and Fechner, U. (2005). Computer-based de novo design of drug-like molecules. Nat Rev Drug Discov 4, 649-663.

Schuetz, A., Min, J., Antoshenko, T., Wang, C.L., Allali-Hassani, A., Dong, A., Loppnau, P., Vedadi, M., Bochkarev, A., Sternglanz, R., and Plotnikov, A.N. (2007). Structural basis of inhibition of the human $\mathrm{NAD}^{+}$-dependent deacetylase SIRT5 by suramin. Structure 15, 377-389.

Suenkel, B., Fischer, F., and Steegborn, C. (2013). Inhibition of the human deacylase Sirtuin 5 by the indole GW5074. Bioorg Med Chem Lett 23, 143-146.

Suri, C., and Naik, P.K. (2015). Combined molecular dynamics and continuum solvent approaches (MM-PBSA/GBSA) to predict noscapinoid binding to g-tubulin dimer. SAR QSAR Environ Res 26, 507-519.

Szczepankiewicz, B.G., Dai, H., Koppetsch, K.J., Qian, D., Jiang, F., Mao, C., and Perni, R.B. (2012). Synthesis of carba-NAD and the structures of its ternary complexes with SIRT3 and SIRT5. J Org Chem 77, 7319-7329.

Talamas, F.X., Ao-Ieong, G., Brameld, K.A., Chin, E., de Vicente, J., Dunn, J.P., Ghate, M., Giannetti, A.M., Harris, S.F., Labadie, S.S., Leveque, V., Li, J., Lui, A.S.T., McCaleb, K.L., Nájera, I., Schoenfeld, R.C., Wang, B., and Wong, A. (2013). De novo fragment design: a medicinal chemistry approach to fragment-based lead generation. J Med Chem 56, 3115-3119.

Tan, M., Peng, C., Anderson, K.A., Chhoy, P., Xie, Z., Dai, L., Park, J., Chen, Y., Huang, H., Zhang, Y., Ro, J., Wagner, G.R., Green, M.F., Madsen, A.S., Schmiesing, J., Peterson, B.S., Xu, G., Ilkayeva, O.R., Muehlbauer, M.J., Braulke, T., Mühlhausen, C., Backos, D.S., Olsen, C.A., McGuire, P.J., Pletcher, S.D., Lombard, D.B., Hirschey, M.D., and Zhao, Y. (2014). Lysine glutarylation is a protein posttranslational modification regulated by SIRT5. Cell Metab 19, 605-617.

Yang, L.L., Li, G.B., Yan, H.X., Sun, Q.Z., Ma, S., Ji, P., Wang, Z.R., Feng, S., Zou, J., and Yang, S.Y. (2012). Discovery of N6-phenyl-1H-pyrazolo[3,4-d]pyrimidine-3,6-diamine derivatives as novel CK1 inhibitors using common-feature pharmacophore model based virtual screening and hit-to-lead optimization. Eur J Med Chem 56, 30-38.

Yang, S.Y. (2010). Pharmacophore modeling and applications in drug discovery: challenges and recent advances. Drug Discov Today 15, 444-450.

Yang, X., Liu, B.Y., Zhu, W.G., and Luo, J.Y. (2015). SIRT5, functions in cellular metabolism with a multiple enzymatic activities. Sci China Life Sci 58, 912-914.

Zang, W., Hao, Y., Wang, Z., and Zheng, W. (2015). Novel thiourea-based sirtuin inhibitory warheads. Bioorg Med Chem Lett 25, 3319-3324.

Zhang, Y., Bharathi, S.S., Rardin, M.J., Uppala, R., Verdin, E., Gibson, B.W., Goetzman, E.S., and Makishima, M. (2015). SIRT3 and SIRT5 regulate the enzyme activity and cardiolipin binding of very long-chain Acyl-CoA dehydrogenase. PLoS ONE 10, e0122297.

Zhou, Y., Zhang, H., He, B., Du, J., Lin, H., Cerione, R.A., and Hao, Q. (2012). The bicyclic intermediate structure provides insights into the desuccinylation mechanism of human sirtuin 5 (SIRT5). J Biol Chem 287, 28307-28314.

Open Access This article is distributed under the terms of the Creative Commons Attribution License which permits any use, distribution, and reproduction in any medium, provided the original author(s) and source are credited. 